\title{
Analysis on the Spreading Characteristics and Innovation of the Intelligence-Competing Program: Who's Still Standing
}

\author{
Xiao Chen
}

Pingxiang University, Pingxiang, Jiangxi, 337000

Key words: Who's Standing Still; spreading characteristic; innovation analysis

\begin{abstract}
In recent yeas, the intelligence-competing programs have been developing vigorously, which not only rose the whole level of Chinese entertainment programs but broadened the horizon of the audience. The essay will take the program Who's Still Standing which is launched by Jiangsu $\mathrm{TV}$ as an example and in the perspective of the spreading characteristics of intelligence-competing entertainment program to analyze the innovation characteristics of Who’s Standing Still.
\end{abstract}

\section{Introduction}

In recent years, the intelligence-competing programs have been developing vigorously. Who's Still Standing is a new intelligence-competing program launched by Jiangsu TV, the rules of which is the competition among contestants with various occupation and labels in limited time in a one- to-one model.Since the premiere of this program in March 2th, 2012, it gained great popularity and became a model in intelligence-competing program in China.

\section{The spreading characteristics of Who's Still Standing}

Without the limitation of time in traditional media, the video websites are typically characterized as new medium and more acceptable to people of varied ages. The major audience of WSS(short for Who's Still Standing) is young people, who uses wider ranges of new media than other ages of people.

Using diversified new medium is the main characteristic of the audience. More than $40 \%$ of the audience visit more than 5 kinds of medium, $57 \%$ of the audience visit the internet, television and newspaper simultaneously according to the CCTV research. Being broadcast on the video websites, WSS met the need of using new media for the group of young people, who are mainly college students or white collar that have just entered the society. They are usually under great pressure in study or work, and have little spare time. Compared with the traditional TV media, video websites offer them a very convenient internet platform, which refrain them from the limitation of time and the trouble of watching programs on fix TV channel and fully satisfied their demand.[1]

Usually advertisement such as products, image or outdoor advertisement are paid. But one can establish public platform to promote oneself, according to the marketing mode in weibo, whose effect is no less than the ads invested on TV and almost need no cost.

Trailers and advertisements are posted on weibo by the staff of the WSS program,which can greatly strengthen the interaction between the program and audiences. Such tactic can both spread the program efficiently and raise the attention of public. On the one hand, taking advantage of the new media to establish a public platform and broaden the market enables a direct conversation between the audience and the program, and through which to meet the demand of the audience, 
further more to help the program definite its own market positioning and make adjustment, therefore to minimize the cost of spreading. On the other hand, through the platform of weibo, the program is capable of adjusting which group is the potential user and make a precise marketing accordingly. Also,the purpose of spreading can be obtained through holding some meaningful theme activities to attract fans, triggering their interests so that information and advertisements related to the program will impress them deeply.

Market has been expanded in TV program and mobile game and raised broad attention of smartphone users, which created a new approach for spreading. "Who's Still Standing (PK edition)" is authorized, which is not simply a mobile game but an approach to participate in the program. Another way is to publicize the mobile game in the program, as the game gains more popularity, so does the program.

Compare to other programs, the mode of quiz is more suitable to be adapted as an educational mobile game and this is an appropriate approach for culturing and word-of-mouth marketing. In "Who's Still Standing(PK edition)", players can not only compete with the previous "Ace" but have a chance of participating in the program, such an interaction is more significant than simple communication with audience. As for the culturing marketing, the mobile game is an intelligent game, which is both entertaining and educational and most importantly, plays the role of promoting the program. In this way, reputation will be captured and word-for-mouth marketing achieved through the publicity promoted by the audience or players . WSSdidn't spread the program via commercial performance like other talent show. Mobile game is undoubtedly the best choice in the presumption of making more people involved into the competition of the contestants. Hence, the "Who's Still Standing(PK edition)" is a successful product as a result of attempt to the field of program spreading.[2]

\section{The innovation of WSS}

Intelligence-competing programs such as Millionaire and Quiz Show made an enormous impact, but since then the development of alike program has halted in China. In the mean while the intelligence-competence programs abroad jumped out of the box and made new progress. WSS is produced based on the Who's Still Standing in NBC, whose rules are exciting and capricious, the contest form is to challenge the former winner and answer questions. The contestant standing in the center of the stage challenges another 10 contestants respectively by answering questions in a form of "wheel war". The champion can take all awards otherwise fails to continue answering.

Quintessence should be absorbed, but not simply copy them. Therefore innovations should be made on the basis of imitating. First is the new ideas, then the reform and innovation. Intelligence-competing should not be simply interpreted as "knowledge" or "common sense”, but we should regard "intelligence" as a macro subject which can be extended to comprehensive abilities. Next task on the list is to innovate the contest form, rules of game and content of the program etc. Only in this way can the substantial innovation emerges and develops more vigorously.

It is unlikely for the Chinese programs to develop in a long term merely by depending importing TV programs overseas. Adjustment should be made according to the local culture and circumstance. Playing a role of guiding the correct values and ideology, spreading positive energy with distinct characteristics is what the program should do to a prospective and vigorous future.

As an intelligence-competing program, WSS didn't focus on the public figures, nor prestigious scholars, but comprised of the common but glaring ordinary people, and there's a chance for everyone to come to the stage. Most of the questions asked in the program are not profound, 
occasionally there will be some theoretical questions. WSS is targeted on mass, thus the questions cover entertainment, sports, health, astronomy and geography.

The program didn't put in the air, but interacted with the audience actively through an open platform and take in suggestions from them, which enable audience to take part in the program and make progress together. The transition of such relation between media and the audience stands for the realization of popularization of Chinese TV programs and Chinese culture. More reverence is given to the individual personality as the rapid development of China, put people first is being emphasized in many areas, thus the TV program based on this idea is gaining increasingly attention and being broadly accepted, which is a short cut for the TV media to accommodate the market competition when internet media and mobile phone media are gradually ripe.

In a time when the medium are changing rapidly and the competition among which are fierce, TV programs are required to adjust its tactics and shift its conception. After all, ordinary people are the major audience of TV media, and a high rating means the program should come close to the real life and adapt to the value orientation of audience in new era. And people are more intended to choose the popularized media under such virtuous circle, again stimulates the process of choosing freely and the orientation of popularization, which is the joint choice between media and audience.

The prominent ingredient in this show is the integration of comedy show and competition, distinctive way of questioning and answering in a pleasant atmosphere, which subverts the traditional rigid impression of intelligence-competing programs left on people. WSS expands the knowledge reserve of people in laughter.

Great efforts are made in program setting. First of all, entertainment and hot issues are the main topic of the quiz. Besides, sports and poems are also involved. And questions are not profound in order to cater the education degree of all ages. People are all equaled on the stage, right answer is the only criteria. And the best way to prove oneself is to defeat all the rivals, no matter you are labeled or not. In addition, WSS is more than an intelligence-competing program, but infuses with reality show perfectly. Thus it is essential to choose the contestants, whose performance will effect the rating directly. There are 10 contestants in every episode, and every one of them has their own personality and characteristic that makes them distinctive from each other. Finally, the performance of the hosts and contestants are also an entertaining ingredient, for example, the contestants will say some slogans to cheer him/herself up and blow the rival before competing; husband-and-wife hosts, ridicule and sarcasm among contestants are all attractive to audience. Entertainment is fully merged in this show and endowed it a new life.

\section{The reasons of its success}

It is the characteristics not by chance that lead to the success of WSS, the reason why it is broadly accepted by the audience can be concluded into following reasons. Firstly, the formality. WSS takes a new form that breaks the fixed diagram of traditional intelligence-competing programs and get rid of the shackle of purely academic contests . Secondly, there is definite system of awarding and punishment. The winner will be honored as "Mars” while the loser drop down from the stage and doesn't have the chance to win the prize. Thirdly, it caters the spirit of challenge. Being able to participate in the challenge and prove oneself is attractive enough, let alone there are plentiful prizes for the winner. Fourthly, the PK form can easily creates an circumstance to make audience feel they are on the spot. And they are tended to be drew in the fierce atmosphere.[3] 


\section{Conclusion}

A new entertainment program can gain great popularity with slightest effort, but by no means easy to maintain it according to the precedent development of the intelligence-competing programs in China. The essay analyzes the characteristics of spreading and innovations of WSS. From the content to advertising, the innovation is an important reason for its success, also an essential tacit for its long-term development, meanwhile, it provides a good example for other domestic intelligence-comping program.

\section{References}

[1] Ru xijia, The Development And Future of Chinese Intelligence-Competing Programs,news,and knowledge, Apr, 2003

[2] Guo ying, The Innovation And Development of Intelligence-Competing Programs---Who’s Still Standing, Youth Literator, 2014

[3] Wang shiwen, The Promotion of Inland Intelligence-Competing Programs, Voice and Screen World, Aug, 2010. 